\title{
ONDULATIONS SECONDAIRES EN FRONT D'INTUMESCENCES ET ONDES SOLITAIRES
}

\section{THIRRIOT * ET S. BEDNARCZYK * *}

\section{I. - Avertissement}

Il a déjà été souvent question d'intumescences et d'ondulations secondaires à la tribune de la Société Hydrotechnique de France et dans les colonnes de La Houille Blanche, où parurent d'excellents articles sous la signature de M. Lemoine, M. Serre et plus récemment, MM. Sandover et Taylor.

\section{II. - Brefs rappels des théories}

En ce qui concerne l'étude théorique de ce phénomène, on peut distinguer deux théories principales suivant que l'on considère un mouvement en bloc ou non.

La première théorie fut établie par Barré de SaintVenant. Elle est fondée sur l'hypothèse de lignes de courant rectilignes et parallèles.

La deuxième théorie, présentée par Boussinesq, admet une répartition non uniforme des vitesses dans une section transversale et par voie de conséquence, prend en considération la courbure des lignes de courant.

Dans cette direction, se situe le travail essentiel que publia M. Serre en 1953, dans La Houille Blanche.

La méthode proposée par M. Serre a été adoptée par M. Sandover pour résoudre le problème des

* Maître de conférences, Professeur à I'E.N.S.E.E.H.T

* Ingénieur diplômé de l'Université de Gdansk. ondulations secondaires, mais M. Sandover a développé cette méthode en l'appliquant aux mouvements relatifs. L'étude présentée ci-dessous est fondée sur la théorie de M. Serre et elle s'inspire avec profit des idées et résultats de M. Sandover.

\section{III. - Expression de la quantité de mouvement et de l'énergie spécifique dans une section rectangulaire}

Comme M. Sandover, nous considérons l'écoulement en mouvement relatif par rapport à un système de référence qui accompagne le front de l'intumescence. La vitesse d'entraînement du repère, d'abord supposée uniforme, est la célérité absolue du premier sommet de l'intumescence.

Le débit apparent dans ce mouvement relatif est constant et égal à :

$$
\Omega=\mathrm{BH}|\mathrm{W}|=\mathrm{B}_{0} \mathrm{H}_{0}\left|\mathrm{~W}_{0}\right|=\mathrm{B}_{0} \mathrm{H}_{0}|\mathrm{~V}-\mathrm{C}|
$$

$V$ est la vitesse absolue des molécules d'eau;

W est la vitesse relative;

C est la célérité absolue du premier sommet de l'intumescence.

Dans le plan des coordonnées, $x$, $z$, se déplaçant avec la célérité d'onde $\mathrm{C}$, dont l'origine est confondue avec le point mobile où se produit la variation du débil, la quantité de mouvement par unité de 
largeur et l'énergie spécifique dans une section transversale, sont données par les équations :

$$
\begin{gathered}
\mathrm{M}=\int_{0}^{\mathrm{H}}\left(\frac{p}{\omega}+\frac{\mathrm{W}_{x}^{2}}{g}\right) d \mathrm{Z} \\
\mathrm{E}=\frac{1}{\mathrm{H}} \int_{0}^{\mathrm{H}}\left(Z+\frac{p}{\omega}+\frac{\mathrm{W}_{x^{2}}+\mathrm{W}_{z}^{2}}{2 g}\right) d Z
\end{gathered}
$$

où :

$H$ est le tirant d'eau;

$\omega$ est le poids spécifique;

$g$ accélération due à la pesanteur.

En écoulement plan, l'équation de continuité s'écrit :

$$
\frac{\partial W_{z}}{\partial x}+\frac{\partial W_{z}}{\partial z}=0
$$

La pression $p$ est définie par l'équation différentielle d'Euler.

$$
\frac{1}{\omega} \frac{\partial p}{\partial Z}=-1-\frac{W_{n}}{g} \frac{\partial W_{z}}{\partial X}-\frac{W_{z}}{g} \frac{\partial W_{n}}{\partial Z}
$$

Compte tenu de l'équation de continuité, les composantes longitudinale $W_{x}$ et verticale $W_{z}$ de la vitesse relative $W$ et aussi leurs dérivées partielles vérifient les relations suivantes:

$$
\begin{aligned}
\mathrm{W}_{x} & =\frac{\Omega}{\mathrm{BH}} \\
\mathrm{W}_{z} & =\frac{\Omega}{\mathrm{BH}^{2}} \mathrm{Z} \frac{d \mathrm{H}}{d \mathrm{X}} \\
\frac{\partial \mathrm{W}_{z}}{\partial \mathrm{X}} & =\frac{\Omega Z}{\mathrm{BH}^{3}}\left[\mathrm{H} \frac{d^{2} \mathrm{H}}{d \mathrm{X}^{2}}-2\left(\frac{d \mathrm{H}}{d x}\right)^{2}\right] \\
\frac{\partial \mathrm{W}_{z}}{\partial z} & =\frac{\Omega}{\mathrm{BH}^{2}} \frac{d \mathrm{H}}{d \mathrm{X}}
\end{aligned}
$$

L'intégration des formules (1), (2) et (3) donne les expressions de la quantité de mouvement et de l'énergie spécifique [5].

$$
\mathrm{M}=\frac{\mathrm{H}^{2}}{2}+\frac{\Omega^{2}}{g \mathrm{~B}^{2} \mathrm{H}}+\frac{\Omega^{2}}{3 g \mathrm{~B}^{2} \mathrm{H}}\left[\mathrm{H} \frac{d^{2} \mathrm{H}}{d \mathrm{X}^{2}}-\left(\frac{d \mathrm{H}}{d \mathrm{X}}\right)^{2}\right]
$$

$$
\mathrm{E}=\mathrm{H}+\frac{\Omega^{2}}{2 g \mathrm{~B}^{2} \mathrm{H}^{2}}+\frac{\Omega^{2}}{6 g \mathrm{~B}^{2} \mathrm{H}^{2}}\left[2 \mathrm{H} \frac{d^{2} \mathrm{H}}{d \mathrm{X}^{2}}-\left(\frac{d \mathrm{H}}{d \mathrm{X}}\right)^{2}\right]
$$

\section{IV. - Equations différentielles du profil d'onde en l'absence de pertes de charge}

Equation DIFFÉRENTIELLE DU PROFIL D'ONDE EN APPLIQUANT LE THÉORÈM DE QUANTITÉ DE MOUVEMENT :

Par hypothèse, nous admettons que les effets du frottement et de la pente du canal se compensent ou que leur somme est négligeable.

Puisque dans la section initiale :

$$
\mathrm{H}=\mathrm{H}_{0} ; \quad \frac{d \mathrm{H}}{d \mathrm{X}}=0 \quad \text { et } \frac{d^{2} \mathrm{H}}{d \mathrm{X}^{2}}=0
$$

la conservation de la quantité de mouvement exprimée par la relation (5), conduit à l'équation :

$$
\begin{aligned}
\frac{\Omega^{2}}{3 g \mathrm{~B}^{2} \mathrm{H}}\left[\mathrm{H} \frac{d^{2} \mathrm{H}}{d \mathrm{X}^{2}}\right. & \left.-\left(\frac{d \mathrm{X}}{d \mathrm{H}}\right)^{2}\right]+ \\
& +\frac{\mathrm{H}^{2}}{2}-\frac{\mathrm{H}_{0}{ }^{2}}{2}+\frac{\Omega^{2}}{g \mathrm{~B}^{2} \mathrm{H}}-\frac{\Omega^{2}}{g \mathrm{~B}^{2} \mathrm{H}_{0}}=0
\end{aligned}
$$

En introduisant le carré du nombre de Froude en mouvement relatif :

$$
\varphi_{0}^{2}=\frac{\Omega^{2}}{g \mathrm{~B}^{2} \mathrm{H}_{0}^{3}}
$$

après transformation, nous obtenons l'équation différentielle suivante :

$$
\begin{aligned}
\mathrm{H} \frac{d^{2} \mathrm{H}}{d \mathrm{X}^{2}}-\left(\frac{d \mathrm{H}}{d \mathrm{X}}\right)^{2} & +\frac{3}{2 \varphi_{0}^{2}} \frac{\mathrm{H}^{3}}{\mathrm{H}_{0}^{3}}- \\
& -3\left(\frac{1}{2 \varphi_{0}^{2}}+1\right) \frac{\mathrm{H}}{\mathrm{H}_{0}}+3=0
\end{aligned}
$$

La relation ci-dessus peut se ramener à une équation différentielle du premier ordre, si nous posons :

$$
\left(\frac{d \mathrm{H}}{d \mathrm{X}}\right)^{2}=u(\mathrm{H})
$$

Ainsi, il vient :

$$
\begin{aligned}
\frac{1}{2} \mathrm{H} \frac{d \mathrm{U}}{d \mathrm{H}}-\mathrm{U}+\frac{3}{2 \varphi_{0}{ }^{2}} \frac{\mathrm{H}^{3}}{\mathrm{H}_{0}{ }^{3}}- & \\
& -3\left(\frac{1}{2 \varphi_{0}{ }^{2}}+1\right) \frac{\mathrm{H}}{\mathrm{H}_{0}}+3=0
\end{aligned}
$$

La solution de cette équation différentielle est obtenue assez simplement, sous la forme d'un développement en série, suivant les puissances croissantes de $\mathrm{H}$ :

$$
\mathrm{U}=\mathrm{C}_{0}+\mathrm{C}_{1} \mathrm{H}+\mathrm{C}_{2} \mathrm{H}^{2}+\mathrm{C}_{3} \mathrm{H}^{3}+\ldots+\mathrm{C}_{n} \mathrm{H}^{n}
$$

où : $\mathrm{C}_{0} ; \mathrm{C}_{1} ; \mathrm{C}_{2} ; \mathrm{C}_{3} ; \ldots \mathrm{C}_{n}$ sont les constantes qui sont déterminées par identification.

En remplaçant dans l'équation de départ $U$ et $d \mathrm{U} / d \mathrm{H}$ par leurs expressions, nous obtenons :

$$
\mathrm{C}_{0}=3 \quad \mathrm{C}_{1}=-\frac{6}{\mathrm{H}_{0}}\left(\frac{1}{2 \varphi_{0}{ }^{2}}+1\right) \quad \mathrm{C}_{3}=-\frac{3}{\varphi_{0}{ }^{2} \mathrm{H}_{0}{ }^{3}}
$$

Tous les coefficients d'ordre supérieur à 3 sont nuls. En conséquence,

$$
\begin{aligned}
\mathrm{U}=\left(\frac{d \mathrm{H}}{d \mathrm{X}}\right)^{2}=-\frac{3}{\varphi_{0}{ }^{2}} \frac{\mathrm{H}^{3}}{\mathrm{H}_{0}{ }^{3}}+ & \\
& +\mathrm{C}_{2} \mathrm{H}^{2}-6\left(\frac{1}{2 \varphi_{0}{ }^{2}}+1\right) \frac{\mathrm{H}}{\mathrm{H}_{0}}+3
\end{aligned}
$$

La constante d'intégration $C_{2}$ sera déterminée par les conditions aux limites qui impliquent pour :

$$
\mathrm{H}=\mathrm{H}_{0} \quad \frac{d \mathrm{H}}{d \mathrm{X}}=0
$$




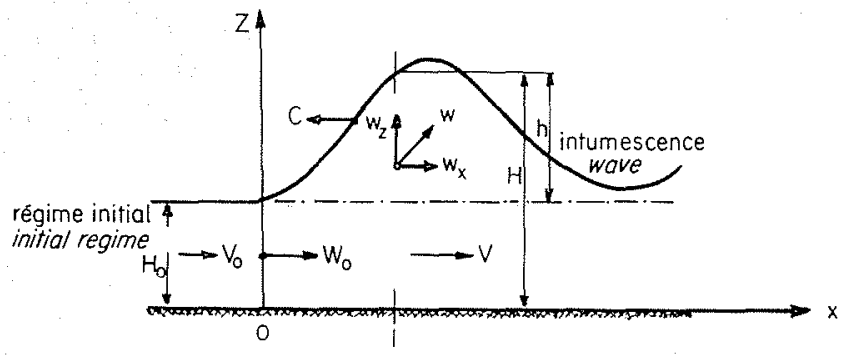

I/

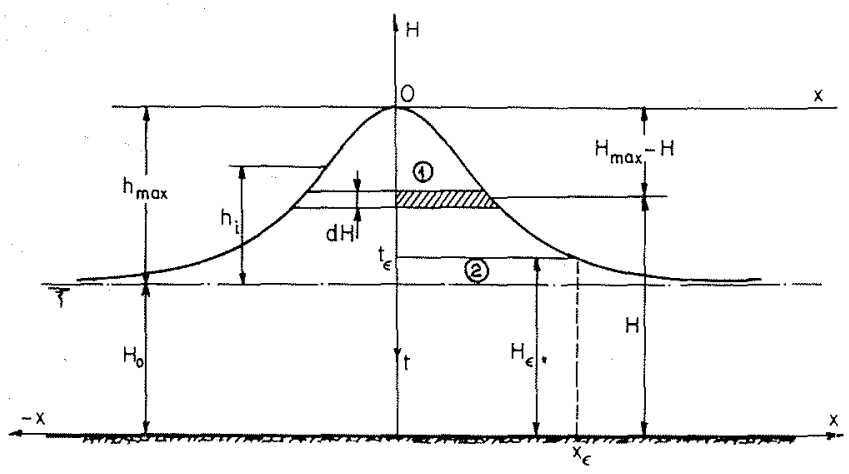

2/

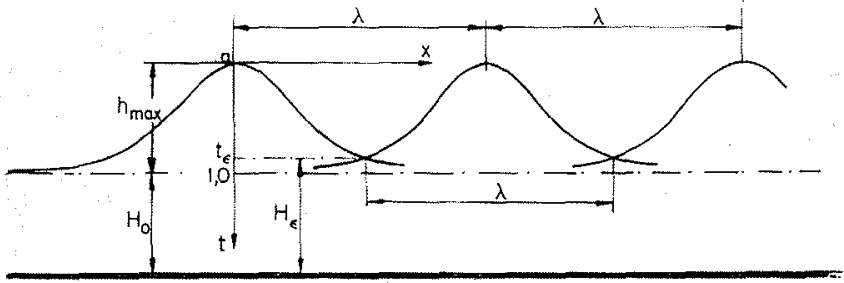

3/

La pente du profil de l'intumescence est donc fixée par l'équation différentielle du premier ordre :

$\left(\frac{d \mathrm{H}}{d \mathrm{X}}\right)^{2}=-\frac{3}{\varphi_{0}^{2}}\left(\frac{\mathrm{H}}{\mathrm{H}_{0}}\right)^{3}+$

$$
+3\left(\frac{2}{\varphi_{0}^{2}}+1\right)\left(\frac{H}{H_{0}}\right)^{2}-3\left(\frac{1}{\varphi_{0}^{2}}+1\right) \frac{H}{H_{0}}+3
$$

ou :

$$
\frac{d \mathrm{H}}{d \mathrm{X}}= \pm \frac{\sqrt{3}}{\varphi_{0}} \sqrt{\varphi_{0}^{2}-\frac{\mathrm{H}}{\mathrm{H}_{0}}}\left(\frac{\mathrm{H}}{\mathrm{H}_{0}}-1\right)
$$

Equation Diffémentielle DU PROFIL D'ONDE EN APPLIQUANT LA CONSERVATION D’ÉNERGIE SPÉCIFIQUE :

En supposant que les effets des pertes de charge sont compensés par l'influence de la pente du radier, l'énergie spécifique ne varie pas le long du profil d'onde :

$$
\mathrm{E}-\mathrm{E}_{0}=0
$$

Ceci conduit à l'équation différentielle :

$$
\begin{array}{r}
\frac{\Omega^{2}}{6 g \mathrm{~B}^{2} \mathrm{H}^{2}}\left[2 \mathrm{H} \frac{d^{2} \mathrm{H}}{d \mathrm{X}^{2}}-\left(\frac{d \mathrm{H}}{d \mathrm{X}}\right)^{2}\right]+ \\
+\mathrm{H}-\mathrm{H}_{0}+\frac{\Omega^{2}}{2 g \mathrm{~B}^{2} \mathrm{H}^{2}}-\frac{\Omega^{2}}{2 g \mathrm{~B}^{2} \mathrm{H}_{0}^{2}}=0
\end{array}
$$

Après intégration, on obtient pour le profil de l'onde la même équation que celle fournie par l'application du théorème d'Euler.

L'intégrale donnant $\mathrm{X}$ en fonction de $\mathrm{H}$ est divergente pour $\mathrm{H}=\mathrm{H}_{0}$. Ainsi, le raccordement du profil de l'ondulation avec le plan d'eau initial a lieu à distance infinie. Le profil défini par la relation ci-dessus est donc celui d'une onde solitaire.

En réalité, l'influence de perte de charge, de pente $d u$ fond et d'accélération du front d'onde transforme cette onde solitaire en train d'ondulations dont le raccordement avec le plan d'eau initial a lieu à distance $d$ finie.

\section{V. - Grandeurs caractéristiques de l'onde solitaire Equations du profil}

Dans le système de coordonnées dont l'origine admet même abscisse que le sommet de l'onde, l'équation du profil, est :

$$
\mathrm{X}=\frac{\varphi_{0} \mathrm{H}_{0}{ }^{3 / 2}}{\sqrt{3}} \int_{\mathrm{H}}^{\mathrm{H}_{\max }} \frac{d \mathrm{H}}{\sqrt{\varphi_{0}{ }^{2} \mathrm{H}_{0}}-\mathrm{H}^{-}\left(\mathrm{H}-\mathrm{H}_{0}\right)}
$$

Après intégration facilitée par le changement de variable :

nous obtenons :

$$
\mathrm{U}=\sqrt{\varphi_{0}{ }^{2}-\frac{\mathrm{H}}{\mathrm{H}_{0}}},
$$

$$
X=\frac{2 \varphi_{0} H_{0}}{\sqrt{3\left(\varphi_{0}^{2}-1\right)}} \operatorname{Arg} \text { th } \sqrt{\frac{\varphi_{0}^{2}-\left(H / H_{0}\right)}{\varphi_{0}^{2}-1}}
$$

La variable réduite :

$$
t=\sqrt{\frac{\varphi_{0}^{2}-\left(\mathrm{H} / \mathrm{H}_{0}\right)}{\varphi_{0}^{2}-1}}
$$

sera très utile pour caractériser les ondulations secondaires.

L'expression (13) permet de tracer un abaque donnant en variables réduites :

$$
h=\frac{\mathrm{H}}{\mathrm{H}_{0}} \text { et } x=\frac{\mathrm{X}}{\mathrm{H}_{0}} \text {, }
$$

la forme des profils correspondant à tout nombre de Froude $\varphi_{0}$ en mouvement relatif.

SURÉlévation Maximale:

Les surélévations extrêmes seront déterminées par la condition :

$$
\frac{d \mathrm{H}}{d \mathrm{X}}=0 \text { soit }\left(\mathrm{H}-\mathrm{H}_{0}\right) \sqrt{\varphi_{0}^{2} \mathrm{H}_{0}-\mathrm{H}}=0
$$

La hauteur maximale a pour expression :

$$
\mathrm{H}_{\max }=\varphi_{0}^{2} \mathrm{H}_{0}
$$

La surélévation maximale au-dessus du plan d'eau initial a donc pour valeur :

$$
h_{\max .}=\mathrm{H}_{\max .}-\mathrm{H}_{0}=\left(\varphi_{0}^{2}-1\right) \mathrm{H}_{0}
$$


VOLUMe DE L'ONDE SOLITAIRE :

Nous nous intéresserons au volume $\mathrm{V}$ de l'onde solitaire compris entre les abscisses - $\mathrm{X}$ et $+\mathrm{X}$ comptées à partir de l'abscisse du sommet prise comme origine.

Ce volume est la somme des volumes $V_{1}$ et $V_{2}$ indiqués sur la figure dont les expressions sont:

$$
\begin{aligned}
& \mathrm{V}_{1}=2 \int_{\mathrm{II}}^{\mathrm{H}_{\max .}} \mathrm{XdH} \\
& \mathrm{V}_{2}=2 \mathrm{X}\left(\mathrm{H}-\mathrm{H}_{0}\right)
\end{aligned}
$$

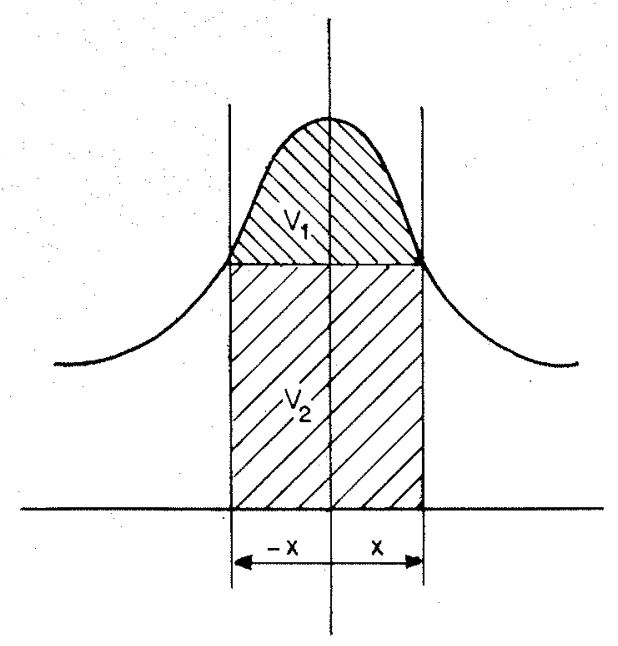

Utilisons la variable réduite $t$ telle que :

Puisque :

$$
t^{2}=\frac{\varphi_{0}{ }^{2}-\left(\mathrm{H} / \mathrm{H}_{0}\right)}{\varphi_{0}{ }^{2}-1}
$$

il vient :

$$
\mathrm{X}=\frac{2 \varphi_{0} \mathrm{H}_{0}}{\sqrt{3\left(\varphi_{0}{ }^{2}-1\right)}} \operatorname{Arg} \operatorname{th} t
$$

$$
\mathrm{V}_{1}=\frac{4 \mathrm{H}_{0}{ }^{2} \varphi_{0} \sqrt{\varphi_{0}{ }^{2}-1}}{\sqrt{3}}\left[t+\left(t^{2}-1\right) \operatorname{Arg} \operatorname{th} t\right]
$$

D'autre part :

$$
V_{2}=\frac{4 H_{0}{ }^{2} \varphi_{0} \sqrt{\varphi_{0}{ }^{2}-1}}{\sqrt{3}}\left(1-t^{2}\right) \operatorname{Arg} \text { th } t
$$

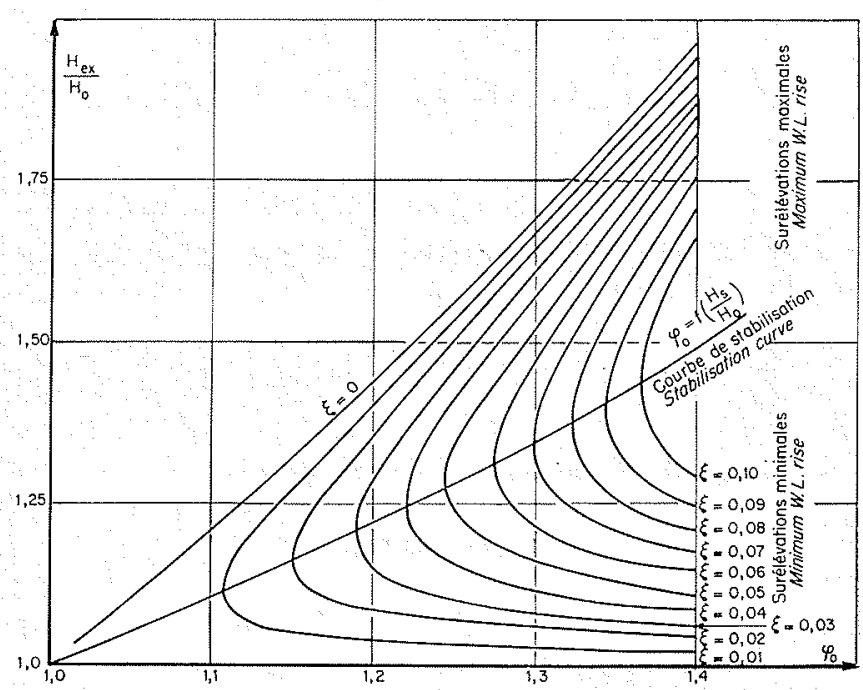

4/ Abaque (Graph for) $\varphi_{0}=f\left(\mathrm{H}_{\mathrm{q} x} / \mathrm{H}_{0}\right)$.
D'où :

$$
\mathrm{V}=\frac{4 \mathrm{H}_{0}{ }^{2} \varphi_{0}}{\sqrt{\varphi_{0}{ }^{2}-1}} t
$$

Cette formule, particulièrement simple, montre que le volume total de l'onde correspondant à $t=1$, a pour valeur :

$$
\mathrm{V}_{0}=2,31 \varphi_{0} \sqrt{\varphi_{0}{ }^{2}-1} \mathrm{H}_{0}{ }^{2}
$$

Forme du profil et résultat du calcul montrent qu'à partir d'une certaine abscisse $X_{\epsilon}$ (ou audessous d'un tirant d'eau $\mathrm{H}_{\epsilon}$ ) le volume de l'onde diffère très peu du volume total.

\section{VI. - Grandeurs caractéristiques de l'ondulation secondaire}

\section{$1^{\circ}$ Hypothèse relative a la formation} DES ONDULATIONS SECONDAIRES :

Pour obtenir les grandeurs caractéristiques de l'ondulation secondaire, nous émettrons quelques hypothèses, dont la témérité sera atténuée par la confrontation avec l'expérience.

Nous supposerons que les ondulations secondaires sont formées par un train d'ondes solitaires, qui se suivent à distance finie.

L'intersection de ces ondes a lieu pour une hauteur d'eau $\mathrm{H}_{\epsilon}$ très voisine de $\mathrm{H}_{0}$ mais cependant légèrement supérieure (fig. 4).

Reste maintenant à fixer la distance entre deux sommets d'ondulation consécutifs, e'est-à-dire la longueur d'onde. La connaissance de la variation de débit qui engendre l'onde, l'expression du volume localisé dans l'onde solitaire et une loi de célérité pourraient permettre la détermination de la longueur d'onde.

Nous avons préféré utiliser une méthode semiempirique. La compilation des résultats expérimentaux présentés par plusieurs auteur's montre que lorsque l'ondulation est bien développée, la longueur d'onde est telle qu'il lui correspond une valeur du paramètre réduit, approximativement constante et égale à $t_{\epsilon}=0,956$.

D'après l'expression de l'abscisse $\mathrm{x}$, on déduit l'évaluation de la longueur d'onde.

$$
\lambda=\frac{4 \varphi_{0}}{\left.\sqrt{3\left(\varphi_{0}\right.}{ }^{2}-1\right)} \operatorname{Arg} \text { th } t_{\epsilon}
$$

et en introduisant la valeur de $t_{\epsilon}$ correspondant à l'onde bien épanouie :

$$
\frac{\lambda}{\mathrm{H}_{0}}=4,41 \frac{\varphi_{0}}{\sqrt{\varphi_{0}^{2}-1}}
$$

Il découle de cette expression que la longueur d'onde devient très grande lorsque $\varphi_{0}$ tend vers l'unité, c'est-à-dire lorsque la variation de hauteur est faible.

Cette remarque explique peut-être pourquoi H. Favre, dans son étude magistrale sur les fronts d'intumescences, annonçait que l'oscillation secondaire disparaissait lorsque la surélévation est faible. En effet, l'ondulation ayant une très grande 
longueur d'onde, le phénomène est difficile à observer et il est perturbé par la modification d'écoulement en corps d'onde.

\section{$2^{\circ}$ Hauteur moyenne d'intumescence :}

Nous définirons la hauteur moyenne d'intumescence comme étant le rapport du volume et de la longueur d'onde :

$$
h_{m}=\frac{V}{\lambda}
$$

Compte tenu des résultats précédents, nous obtenons :

$$
h_{m}=\mathrm{H}_{0}\left(\varphi_{0}^{2}-1\right) \frac{t_{\epsilon}}{\operatorname{Arg} \operatorname{th} t_{\epsilon}}
$$

Si on admet que $t_{\epsilon}=0,956$, il vient :

$$
\eta=\frac{\operatorname{Argth} t_{\epsilon}}{t_{\epsilon}}=2 \quad \text { et } h_{m}=\frac{h_{\max }}{2}
$$

La hauteur moyenne d'intumescence $\mathrm{H}_{m}$ (ou hauteur pondérée) est alors égale à la moyenne des hauteurs extrêmes $H_{0}$ et $H_{\max }$.

$3^{\circ}$ Répartition de DÉbit A L'INTERRIEUR DE L'ONDULATION :

Considérons l'équation de continuité dans le mouvement relatif :

$$
\mathrm{WH}=\mathrm{W}_{0} \mathrm{H}_{0}
$$

soit, par rapport au repère fixe :

d'où :

$$
(\mathrm{V}-\mathrm{C}) \mathrm{H}=\left(\mathrm{V}_{0}-\mathrm{C}\right) \mathrm{H}_{0}
$$

$$
\mathrm{Q}=\mathrm{Q}_{0}+\mathrm{C}\left(\mathrm{H}-\mathrm{H}_{0}\right)
$$

expression habituelle de continuité en front d'intumescence.

Si $q=\mathrm{Q}-\mathrm{Q}_{0}$ est la variation de débit, celle-ci est liée linéairement à la variation de hauteur $h$ puisque $c$, la célérité absolue, est constante.

Lorsque le facteur de forme $\eta$ est égal à 2 (deux) le débit relatif $q$ au sommet de l'onde est le double du débit moyen.

Ainsi, dans le cas d'une onde d'aval due à une fermeture totale, le débit $Q$ dans la section $d u$ sommet d'onde sera négatif. La vitesse négative des molécules d'eau a pu être observée dans notre étude expérimentale.

\section{VII. - Influence des pertes de charge sur les oscillations secondaires}

En mouvement relatif, l'introduction des pertes de charge dans l'équation différentielle générale est asse $z$ délicate, car les pertes par frottements sont toujours de signe opposé à la vitesse absolue des molécules. Ainsi, le signe de J, gradient de la ligne de charge, dépend de $V$ et non de $W$ :

$$
\mathrm{E}_{0}=\mathrm{E}+\int_{0}^{\mathrm{x}} \mathrm{J}(\mathrm{V}, \mathrm{H}) \mathrm{dX}
$$

Dans l'étude de la répartition de débit, nous venons de voir aue la vitesse réelle peut changer de sens à l'intérieur de la masse de l'ondulation. Par conséquent, le long du profil, la perte de charge linéaire $J$ peut changer de sens et l'énergie totale peut décrôttre, puis recroître, en fonction de l'abscisse.
Pour simplifier et pour mener à bien une étude qualitative, nous supposerons que la perte de charge est proportionnelle au carré de la vitesse relative.

La conservation globale de l'énergie conduit alors à l'équation :

$$
\begin{aligned}
& \mathrm{H}+\frac{\Omega^{2}}{6 g \mathrm{~B}_{0}^{2} \mathrm{H}^{2}}\left[2 \mathrm{H} \frac{d^{2} \mathrm{H}}{d \mathrm{X}^{2}}-\left(\frac{d \mathrm{H}}{d \mathrm{X}}\right)^{2}\right]+ \\
& +\frac{\Omega^{2}}{2 g \mathrm{~B}_{0}{ }^{2} \mathrm{H}^{2}}+\int_{0}^{\mathrm{X}} \frac{\mathrm{C}_{f} \mathrm{~W}^{2}}{2 g} \frac{d \mathrm{X}}{\mathrm{H}_{0}}=\mathrm{H}_{0}+\frac{\mathrm{W}_{0}{ }^{2}}{2 g}
\end{aligned}
$$

$\mathrm{C}_{f}$ est un coefficient approprié de dissipation d'énergie.

Afin de faciliter l'intégration de cette équation, nous poserons encore une hypothèse : nous supposerons que le terme représentant les pertes de charge varie peu le long d'une portion de profil, c'est-à-dire qu'il est pratiquement indépendant de $x$.

L'hypothèse n'est pas mince et elle est très discutable, mais elle permet d'obtenir rapidement des résultats qualitatifs.

Dans ces conditions, les pertes de charge sont exprimées par l'expression :

$$
\frac{\xi w)^{2}}{2 g}
$$

En introduisant encore le carré du nombre de Froude, l'équation différentielle prend la forme :

$$
\begin{aligned}
2 \mathrm{H} \frac{d^{2} \mathrm{H}}{d \mathrm{X}^{2}}-\left(\frac{d \mathrm{H}}{d \mathrm{X}}\right)^{2} & +\frac{6}{\varphi_{0}{ }^{2}} \frac{\mathrm{H}^{3}}{\mathrm{H}_{0}{ }^{3}}- \\
& -3\left(\frac{2}{\varphi_{0}{ }^{2}}+1\right) \frac{\mathrm{H}_{2}}{\mathrm{H}_{0}{ }^{2}}+3(1+\xi)=0
\end{aligned}
$$

L'équation ci-dessus, semblable à celle obtenue en l'absence de perte de charge, peut se ramener à une équation différentielle du premier ordre par rapport à la fonction :

$$
u=\left(\frac{d \mathrm{H}}{d \mathrm{X}}\right)^{2}
$$

On cherche encore la solution sous la forme d'un développement en série suivant les puissances croissantes de $\mathrm{H}$.

II vient.

$\left(\frac{d H}{d X}\right)^{2}=-\frac{3}{\varphi_{0}^{2}} \frac{H^{3}}{H_{0}^{3}}+3\left(\frac{2}{\varphi_{0}^{2}}+1\right)+C_{1} H+3(1+\xi)$

La condition à la limite $(d \mathrm{H} / d \mathrm{X})=0$ pour $\mathrm{H}=\mathrm{H}_{0}$ fixe la valeur de $\mathrm{C}_{1}$ :

$$
\mathrm{C}_{1}=-\frac{3}{\mathrm{H}_{0}}\left(\frac{1}{\varphi_{0}^{2}}+\xi+2\right)
$$

d'où l'équation du profil d'onde :

$$
\begin{array}{r}
\frac{d \mathrm{H}}{d \mathrm{X}}= \pm \sqrt{\frac{3}{\varphi_{0}^{2}}\left(\frac{\mathrm{H}}{\mathrm{H}_{0}}-1\right)-\left[\frac{\mathrm{H}^{2}}{\mathrm{H}^{2}}\right.} \\
+\left(1+\varphi_{0}^{2}\right) \frac{\mathrm{H}}{\mathrm{H}_{0}}-\varphi_{0}^{2}(1+\xi)
\end{array}
$$




\section{SuRÉlÉvatyons EXTRÉmales :}

Les tirants d'eau extrêmes correspondent à $(d \mathrm{H} / d \mathrm{X})=0$, racines de l'équation :

$$
\frac{\mathrm{H}^{2}}{\mathrm{H}_{0}{ }^{2}}-\left(1+\varphi_{0}^{2}\right) \frac{\mathrm{H}}{\mathrm{H}_{0}}+\varphi_{0}^{2}(1+\xi)=0
$$

Introduisons la surélévation $h=\mathrm{H}-\mathbf{H}_{0}$, il vient :

$$
\left(\frac{h}{\mathrm{H}_{0}}\right)^{2}-\left(\varphi_{0}^{2}-1\right) \frac{h}{\mathrm{H}_{0}}+\varphi_{0}^{2 \xi}=0
$$

d'où les surélévations extrêmes :

et :

$$
\frac{h_{\max }}{\mathrm{H}_{0}}=\frac{\varphi_{0}^{2}-1}{2}+\sqrt{\left(\frac{\varphi_{0}^{2}-1}{4}\right)^{2}-\varphi_{0}^{2 \xi}}
$$

$$
\frac{h_{\min .}}{\mathrm{H}_{0}}=\frac{\varphi_{0}{ }^{2}-1}{2}-\sqrt{\left(\frac{\varphi_{0}{ }^{2}-1}{4}\right)^{2}-\varphi_{0}{ }^{2 \xi}}
$$

La moyenne de ces surélévations extrêmes est égale à la moitié de la surélévation maximale en l'absence de perte de charge.

Pour obtenir rapidement les surélévations extrêmes, nous proposons un abaque (fig. 4) qui traduit la relation entre $\varphi_{0}$ et $\mathrm{H}_{\mathrm{ex}}$.

$$
\varphi_{0}=\sqrt{\frac{\mathrm{H}_{\mathrm{ex}}\left(\mathrm{H}_{\mathrm{ex}}-\mathrm{H}_{0}\right)}{\mathrm{H}_{0}\left[\mathrm{H}_{\mathrm{ex}}-(1+\xi) \mathrm{H}_{0}\right]}}
$$

Dans un écoulement, le long de l'ondulation secondaire le nombre de Froude relatif $\varphi_{0}$ reste constant. Donc le lieu des extrêmes successifs sur la figure 5 est une verticale qui coupe les courbes correspondant à différentes valeurs de $\xi$ coefficient de perte de charge.

Supposons que la perte d'énergie croisse avec l'abscisse; donc les extrêmes successifs vont correspondre à des valeurs croissantes de $\xi$. Ainsi, l'amplitude entre sommet et creux va décroître avec l'élongation $X$ jusqu'à s'annuler pour une valeur de $\xi$ bien définie; on aura alors un tirant d'eau stabilisé (si les conditions d'écoulement ne sont pas trop vite modifiées derrière le front d'onde).

Pour cette surélévation stabilisée, l'équation donnant les hauteurs d'eau extrêmes admet évidemment une racine double, d'où :

$$
\frac{h_{s}}{\mathrm{H}_{0}}=\frac{\varphi_{0}^{2}-1}{2} \text { et } \xi=\frac{\left(\varphi_{0}^{2}-1\right)^{2}}{4 \varphi_{0}^{2}}
$$

soit encore:

$$
\varphi_{0}=\sqrt{1+2 \xi+2 \sqrt{\xi}(1+\xi)}
$$

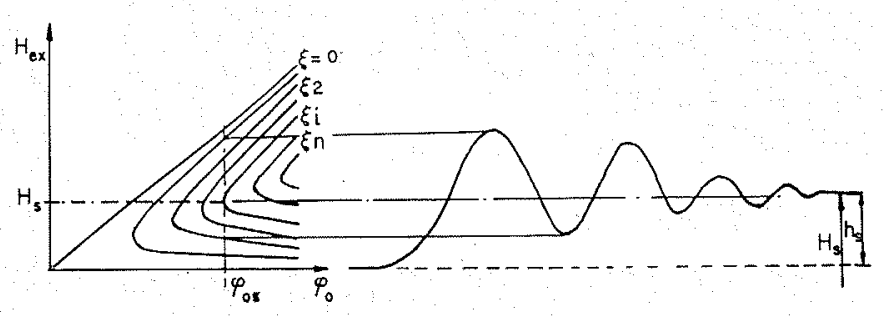

5/
Notons que si la variation de $\xi$ n'est pas monotone ( $J$ peut changer de sens, avons-nous remarqué), les surélévations maximales peuvent croître. C'est un fait d'expérience que le sommet le plus haut ne correspond pas toujours à la première oscillation. En réalité, ceci n'est pas uniquement dû à l'influence de la perte de charge, mais aussi à l'effet conjugué de la pente du fond et d'une accélération du front d'intumescences qui font introduire dans l'équation différentielle des termes complémentaires semblables au terme de perte de charge.

Profil de L'ONDULATION SECONDAIRE :

Pour tracer rapidement le profil de l'ondulation, on peut utiliser une méthode approchée fondée sur les résultats précédents.

Supposons avoir représenté les courbes intégrales de l'équation du premier ordre correspondant à différentes valeurs du paramètre $\xi$.

En faisant croître la perte de charge par palier, nous pouvons pour chacun de ces paliers emprunter la portion de profil correspondante sur l'abaque des courbes intégrales.

La construction de l'abaque mentionné ci-dessus nécessite l'intégration de l'équation différentielle:

$$
\frac{d \mathrm{H}}{d \mathrm{X}}= \pm \sqrt{\frac{3}{\varphi_{0}^{2}}\left(\frac{\mathrm{H}}{\mathrm{H}_{0}}-1\right)\left[\begin{array}{l}
\frac{\mathrm{H}_{2}}{\mathrm{H}_{0}^{2}}+\left(1+\varphi_{0}^{2}\right) \\
\left.\times \frac{\mathrm{H}}{\mathrm{H}_{0}}-\varphi_{0}^{2}(1+\xi)\right]
\end{array}\right.}
$$

Des changements de variables successifs conduisent à une intégrale elliptique.

\section{VIII. - Etude expérimentale}

Programme D'essais :

Pour juger de la validité de nos hypothèses et vérifier les résultats de notre étude théorique simplifiée, nous avons effectué une étude expérimentale systématique.

Les essais exécutés concernent essentiellement les ondes positives d'aval provoquées par la fermeture instantanée d'une vanne à l'extrémité d'un canal vitré.

Comme paramètres caractéristiques des essais, nous avons retenu le nombre de Froude $\mathscr{F}_{0}$ et le tirant moyen de l'écoulement initial en régime permanent.

Nous avons considéré les valeurs suivantes:

$$
\begin{array}{llll}
\mathscr{Y}_{0}=0,10 & 0,20 & 0,30 & \text { et } 0,40 \\
\mathrm{H}_{0}=0,10 \mathrm{~m} & 0,20 \mathrm{~m} & 0,30 \mathrm{~m}
\end{array}
$$

Avec les essais de fidélité, le nombre d'expériences effectuées dépasse la quinzaine.

\section{DESCRIPTION DE L'INSTALLATION D'EXPERIENCE :}

L’ensemble des installations est représenté sur la figure 6 .

La pompe pouvant donner un débit total de $200 \mathrm{l} / \mathrm{s}$ environ, alimente un réservoir à niveau constant. 
Le canal, à section rectangulaire, a les dimensions suivantes:

longueur. . . . . . . . . . . . 21,28 m

largeur. . . . . . . . . . . . .

hauteur utile. ............ $0,95 \mathrm{~m}$

pente longitudinale........... nulle

ProcédÉ de Mesure :

Mesure du débit:

En régime permanent, le débit est mesuré à la sortie du canal de retour à l'aide d'un déversoir rectangulaire sans contraction installé d'après les normes françaises.

Mesure de tirant d'eau :

En régime transitoire, le niveau est repéré à l'aide de jauges électriques à résistance disposées le long du canal de la façon suivante :

\begin{tabular}{|c|c|}
\hline $\begin{array}{c}\text { NumÉro } \\
\text { DE JAUGE }\end{array}$ & $\begin{array}{c}\text { Distance EN MèTREs } \\
\text { DE L'EXTRÉMITÉ AVAL }\end{array}$ \\
\hline 8 & 0,36 \\
7 & 2,96 \\
6 & 6,01 \\
5 & 9,06 \\
4 & 10,55 \\
3 & 12,11 \\
2 & 15,15 \\
1 & 18,19 \\
\hline
\end{tabular}

Pour mesurer les niveaux en régime permanent et exécuter les étalonnages des capteurs à résistance, nous avons utilisé des pointes associées aux supports des jauges électriques. La position des pointes est repérée sur une échelle permettant approximativement des lectures à un dixième de millimètre.

Les ondulations se développent d'autant plus vite que le nombre de Froude réel est plus élevé. Pour $\mathscr{G}_{0}=0,2$, il semble que l'ondulation s'est épanouie dans tous les cas d'écoulement étudiés.

Le critère de longueur qui intervient est le rapport $\mathrm{L} / \mathrm{H}_{0}$ ( $\mathrm{L}$ étant la longueur du canal). Pour les plus faibles tirants d'eau $\left(\mathrm{H}_{0}=0,10 \mathrm{~m}\right)$ ce rapport atteint 213 et les ondulations sont bien développées.

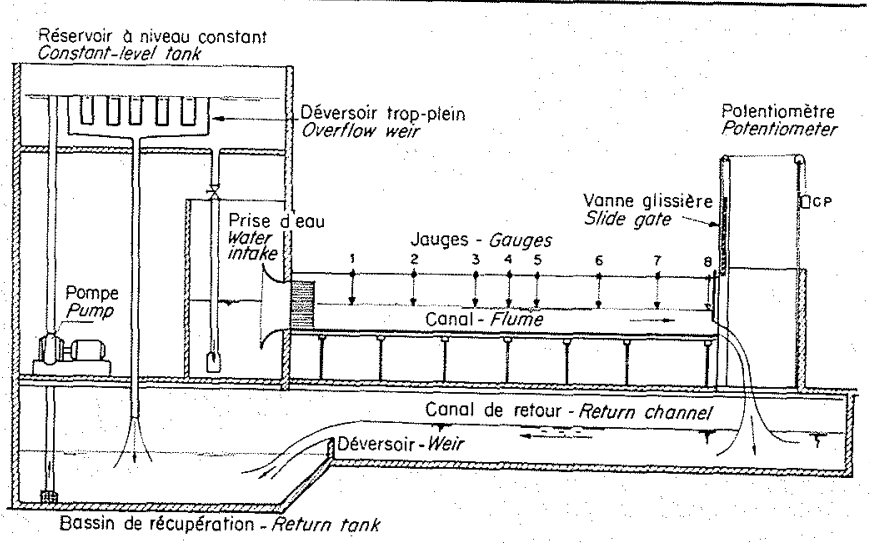

6) Schéma de l'installation.

Experimental layout.

\section{IX. - Comparaison des résultats théoriques et expérimentaux}

Profil D'ONDE :

Les hypothèses à la base de notre étude théorique impliquent que l'ondulation soit pleinement développée, ce qui 'n'est que partiellement vérifié expérimentalement.

Pour effectuer la comparaison, nous avons choisi un écoulement à tirant d'eau initial assez grand $\left(\mathrm{H}_{0}=0,40 \mathrm{~m}\right)$ correspondant à un nombre de Froude de 0,3.

Les figures $n^{\text {os }} 7,8$ et 9 montrent l'évolution du profil expérimental du profil théorique dépendant de la valeur du nombre de Froude relatif $\varphi_{0}$.

La bonne concordance des profils expérimental et théorique à l'extrémité amont montre que l'hypothèse relative à la formation des oscillations secondaires, n'est pas déraisonnable dans le cas où les ondes sont bien développées et lorsque accélération et pertes de charge sont négligeables.

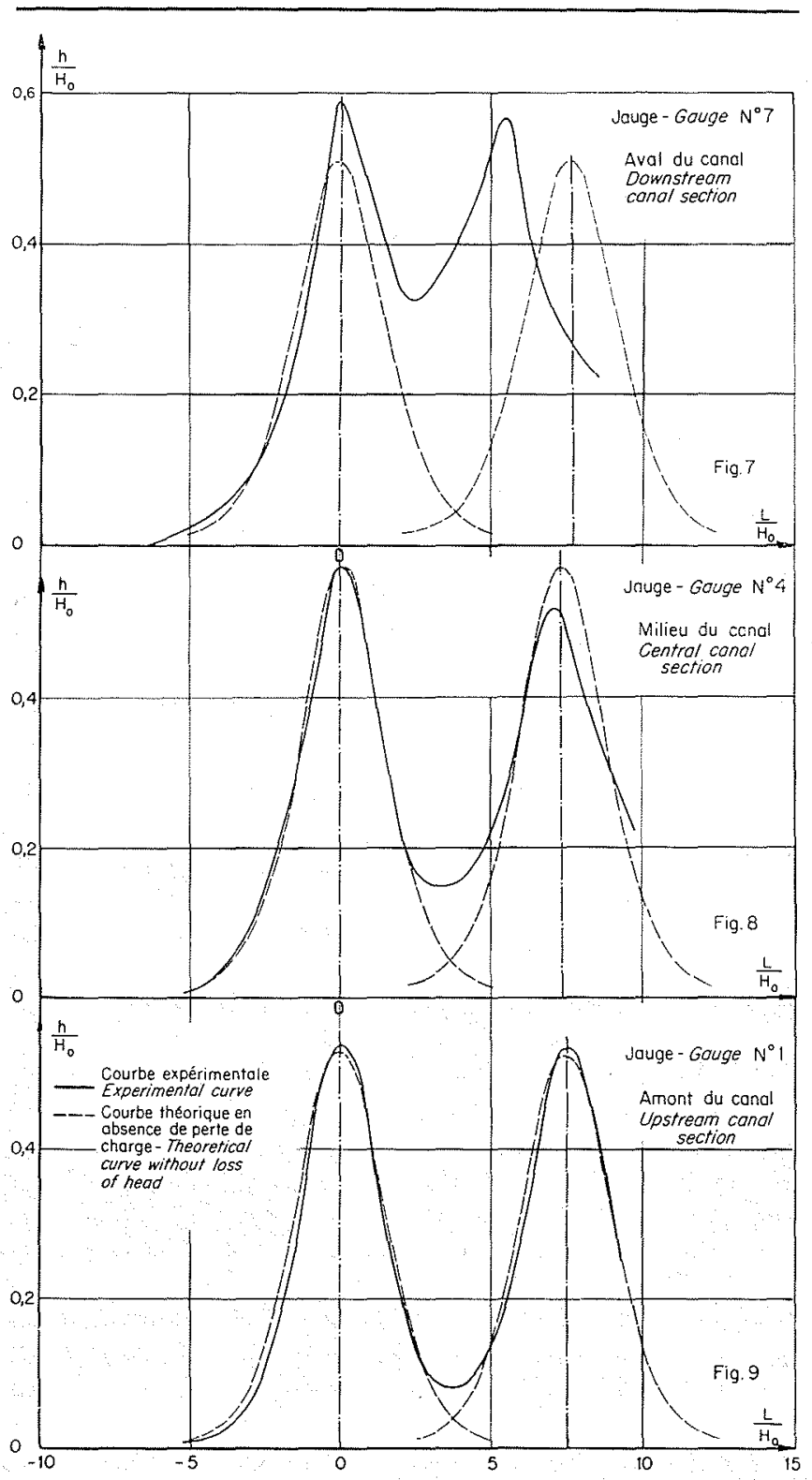

7/8/ ef 9/ Proflis expérimentaux et théoriques. Experimental and theoretical profiles. 
Oscillations MAXimales :

Les résultats expérimentaux représentés sur la figure 10 montrent que la formule proposée est en général satisfaisante.

Cependant, pour les faibles nombres de Froude $\left(\varphi_{0}<1,05\right)$ les valeurs de $H_{\max } / H_{0}$ fournies par l'expérience sont supérieures à celles données par la formule :

$$
\mathrm{H}_{\text {max. }}=\varphi_{0}^{2} \mathrm{H}_{0}
$$

Ceci tient vraisemblablement à l'influence de la répartition verticale de la composante longitudinale des vitesses et à l'effet de l'accélération du front d'onde.

\section{LONGUEUR D'ONDE :}

La figure 11 montre le bon accord existant entre les valeurs de la longueur d'onde mesurée après un certain temps de propagation et les prévisions théoriques.

Cependant, pour les faibles valeurs du nombre de Froude $\varphi_{0}$, la longueur d'onde est limitée même pour une ondulation bien développée. La longueur d'onde réduite, voisine de 8 en moyenne est toujours comprise entre 5 et 10 .

\section{HaUteur moYenne D'INTUMESCENCES :}

Par définition, cette hauteur moyenne est le rapport du volume d'onde par la longueur d'onde. Pour les ondes bien développées, nous trouvons une bonne coïncidence entre les résultats théoriques ef expérimentaux, sauf pour les valeurs du nombre de Froude très voisines de l'unité.

En utilisant les coordonnées proposées par M. Favre, soit $h_{m} / \mathrm{H}_{0}$ et $h_{\max .} / h_{m}$, il apparaît que le rapport entre la hauteur maximale et la hauteur moyenne est d'environ 2, résultat mis en évidence dans notre étude théorique dans le cas de l'onde développée.

\section{X. - Conclusion}

Les ondulations secondaires dépendent à la fois des conditions locales du régime porteur et de leur évolution antérieure au cours de la propagation de l'intumescence.

Il en est un peu des ondulations secondaires comme des personnes; leur comportement, à un certain moment dépend d'une part, de leur éducation ou de leur histoire et d'autre part, des circonstances ou de l'environnement.

En somme, leur attitude dépend de leur degré de retentissement. Pour notre part, nous pensons que les ondulations sont très influençables et qu'elles ont peu de mémoire. Cette opinion est celle sur laquelle se fondent habituellement les chercheurs qui étudient le phénomène d'ondulation pleinement épanouie. Dans ce cas, l'étude expérimentale dont nous venons de parler, montre que les résultats théoriques représentent convenablement la réalité en dépit des hypothèses simplificatrices.

Cependant, les divergences pour les faibles nombres de Froude, la dispersion dans le plan de Favre, montrent que bien des points restent obscurs. Nous avons cherché l'explication par l'influence de la

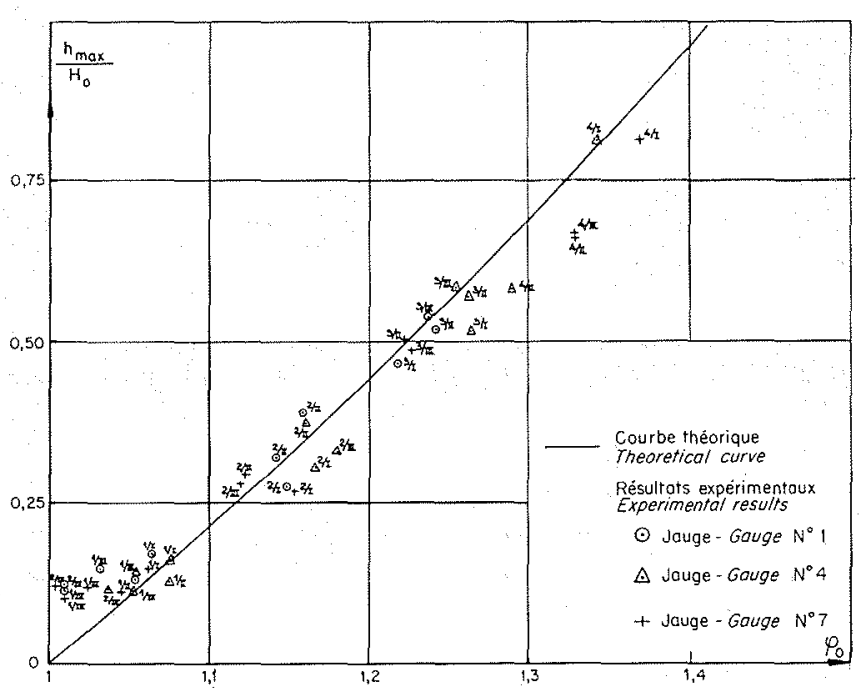

10/ Abaque (Graph for) $h / \mathrm{H}_{0}=f\left(\varphi_{0}\right)$.

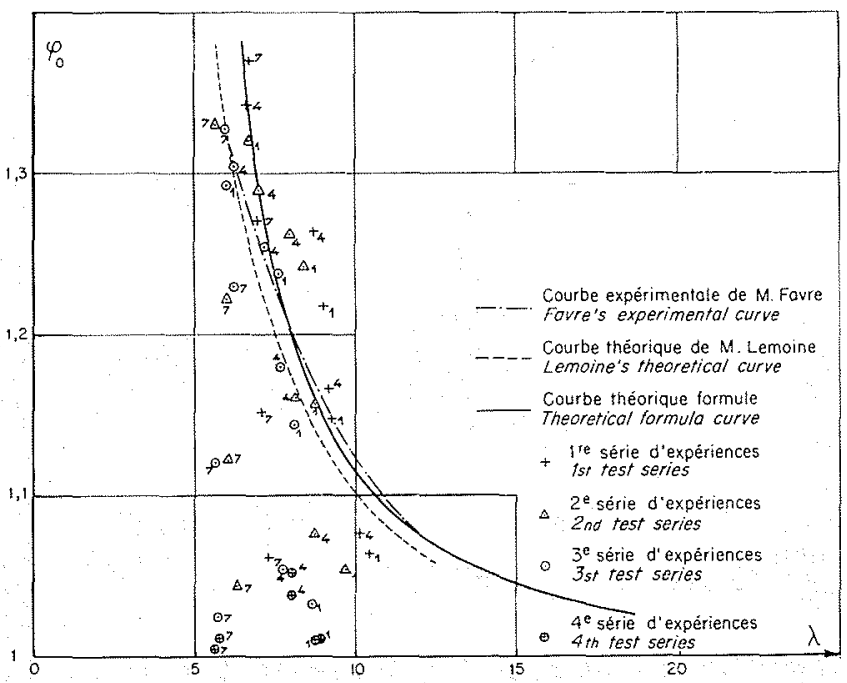

11/ Longueur d'onde en fonction du nombre de Froude. Wave length vs. Froude number.

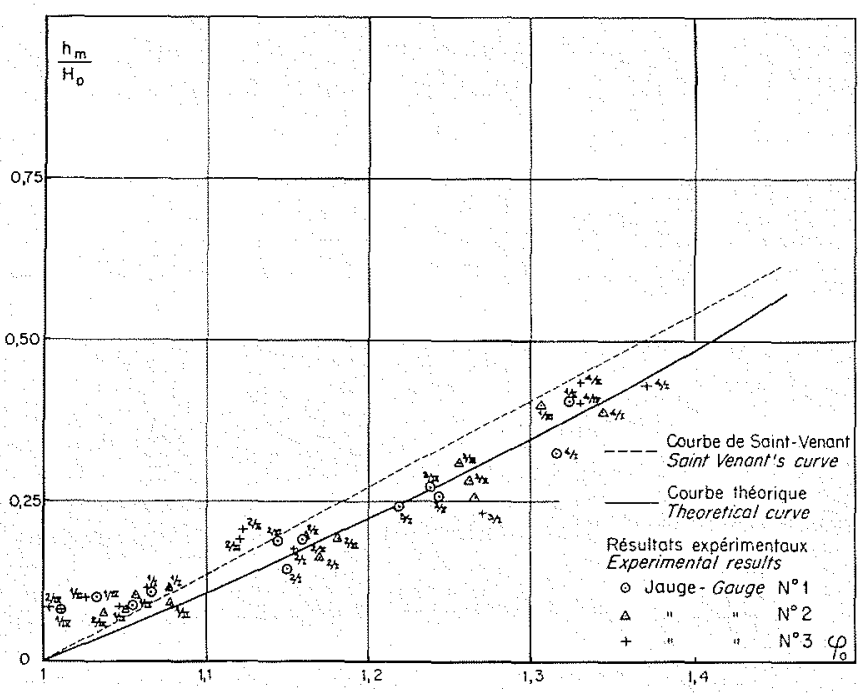

12/ Hauteur d'onde en fonction du nombre de Froude. Wave height vs. Froude number. 
gravité et de l'accélération longitudinale, mais les résultats obtenus, s'ils sont instructifs, ne sont pas décisifs.

De même, une étude similaire a été entreprise dans le cas des canaux à profil trapézoïdal. Les résultats théoriques font apparaître des surélévations maximales relativement moins importantes que dans le cas du profil rectangulaire.

Enfin, des réserves sont à faire en ce qui concerne la validité rigoureuse de la similitude de Froude des fronts d'onde. Même dans le cas de similitude géométrique, il semble qu'il existe un effet d'échelle non négligeable.
Bibliographie

J. SANDover. - An investigation of surge wave profiles in open channels flow. Thèse présentée à l'Université d'Edimbours.

J. Sandoyer et O. Zienkiewicz. - Experiments on surge waves. Water Power, n 11, 1957, p. 418.

J. SANDover et C. TAYLoR. - Les ondes de translation et les ondulations de front d'onde. La Houlle Blanche, $\mathrm{n}^{\circ} 3$ 1963 , p. 443.

F, Serre. - Contribution à l'étude des écoulements permanents et variables dans les canaux. La Houllle Blanche, $\mathrm{n}^{\circ} 3,1953$, p. $374-388 ; \mathrm{n}^{\circ} 6,1953$, p. 830-872.

C. Taylor. - Ondes secondaires se propageant dans les canaux découverts primastiques. Thèse soutenue à l'Université de Wales, août 1960.

\section{Discussion}

\section{Président : M. Chapouthier}

M. le Président remercie $M$. Thin contradietion de terminologie apparaît entre l'« onde solitaire» et les «ondes secondaires》.

M. Thinrot indique que le terme ondulations secondaires $\gg$ vient de la distinction que l'on fait avec l'onde primaire, qui est constituée par l'intumescence elle-même, c'est-à-dire l'onde de translation, l'onde à front raide, telle qu'on l'entend habituellement.

Le front d'intumescence, dans certaines conditions, devient ondulé et l'on appelle généralement ondulations secondaires ce qui se superpose à la hauteur moyenne du front d'intumescence.

D'autre part, M. le Président trouve que le fait de raccorder deux ondes solitaires pour en faire une onde double est un peu hardi.

M. Thmniot ne nie pas la témérité des hypothèses. Il semble toutefois que l'intumescence se fractionne en ondes solitaires, qui conservent quand même leur personnalité ef, pour employer un terme familier, la liberté de chaque onde solitaire est limitée par celle de ses voisines, car, lorsque, an bout d'un certain temps, elles ont trouvé leur équiljpre, ceci signifie qu'elles sont à distance sufísamment granae de leur voisine. A ce moment-là, il n'y a plus de compression.

M. Baxal n'est pas tellement surpris que le profil des ondes secondaires puisse être assez bien représenté par une suite d'ondes solitailes.

Il semble bien en effet que les ondes périodiques en laible profondeur d'amplitude relative importante ne puissent pas avoir une configuration sensiblement différente.

Ceci a été notamment mis en évidence dans un travail de Laitone publié l'année dernière dans un bulletin du Beach Erosion Board.

La méthode utilisée par Laitone consiste à chercher des solutions des équations générales des fluides parfaits à surface libre à deux dimensions qui soient développées par rapport à un petit paramètre représentant non l'amplitude du mouvement comme pour les ondes de surface (étude de Miche notamment), mais le rapport de l'amplitude verticale du mouvement à son amplitude longitudinale.

La première approximation ainsi obtenue est celle de Saint-Venant.

La deuxième approximation donne les ondes périodiques dites cnoidales dont l'onde solitaire est un cas particulier.

L'approximation suivante permet de discuter la validité de ces solutions.

Cette dernière partie de l'étude met en évidence que le calcul en deuxième approximation ne paraît justifié que pour des ondes cnoïdales dont la forme est voisine de celle de la partie centrale d'une onde solitaire.

Il faut rappeler que cette étude n'est valable que pour des intumescences se propageant dans un fluide où la répartition verticale de la vitesse est uniforme, ce qui risque d'être assez loin du cas des ondes secondaires.

M. BENET demande si la comparaison entre les essais théoriques et nature pour des canaux trapézoïdaux a été faite au centre de la section ou au bord, car les différences d'ondulation sont très sensibles.

M. Thurro't rappelle que les essais italiens portaient sur des enregistrements au milieu de la section et que le Laboratoire de Toulouse a aussi les comparaisons dans l'axe du canal. La différence signalée par M. BENET est un phénomène général, qui a fait l'objet de la thèse de Taylor et de diverses observations.

Notamment, notre collègue M. Castex a eu l'occasion, lors d'essais à l'usine de Palaminy, d'observer une cambrure transversale des premières ondulations secondaires qui produisait une flèche de 60 à $70 \mathrm{~cm}$ sur une largeur du canal, qui devait être d'environ $25 \mathrm{~m}$. Plus récemment, ce phénomène a été observé aussi sur le canal d’oraison. En fait, il faudrait plutôt se préoceuper de ce qui se passe sur les bords en raison des risques de débordement.

M. Novgaro fait une remarque relative aux expérienees qui doivent être entreprises pour vérifier les valjdités des hypothèses de répartition des vitesses admises par M. THImRiot.

M. Thminot confirme qu'il a essayé un procédé artisanal de mesure de vitesse qui consiste à faire flotter de petites balles dans le courant (figure) mais les enregistrements sur

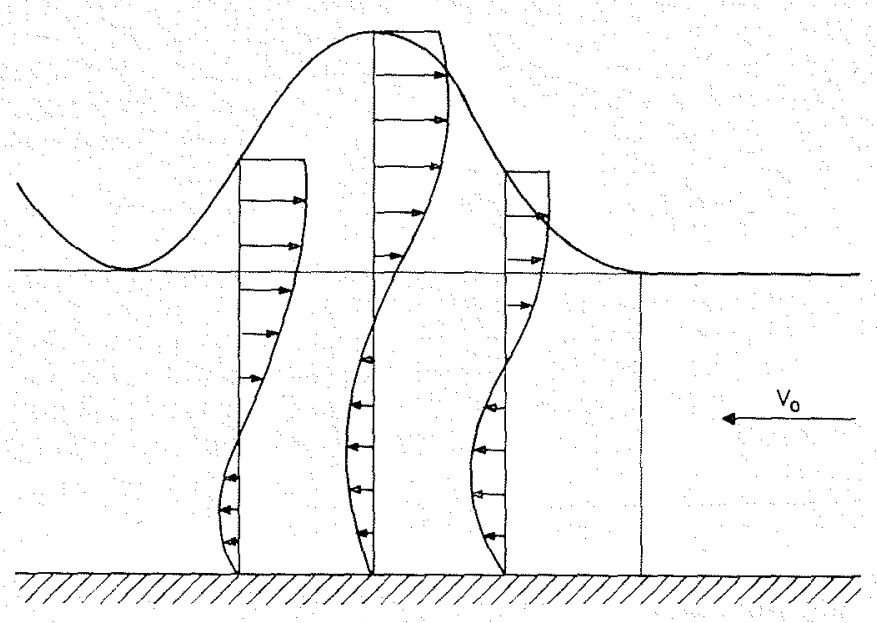




\title{
C. THARIOT et S. BEDNARCZYK
}

films qui ont été réalisés ne sont pas très nets. Néanmoins, on observe très bien les déformations de la répartition des vitesses au passage de l'onde : il semble qu'apparaît rapidement un courant de retour près de la surface, mais, au voisinage $d u$ fond, la vitesse est modifée beaucoup plus lentement.

M. ThinRor croit qu'industriellement pen d'essais ont été réalisés pour déterminer cette répartition des vitesses : il n'a connaissance que d'essais exécutés en Roumanie.

Cette année, le Laboratoire de Toulouse dirige ses efforts sur l'obtention de renseignements sur la répartition des vitesses au moyen de jauges de contrainte fixées sur des capteurs élastiques.

M. ThIRrot sollicite des suggestions pour faire ces observations.

\section{Secondary undulations at a wave front, and solitary waves}

\author{
by $C_{x}$ Thirriot * and S. Bednarczyk * *
}

The results presented in this paper follow the excellent work by Lemoine and Serre and more recent researcl by Sandover and Taylor, an account of which latier has already becn published in La Houille Blanche.

A distinction is made between two principal transient canal flow research theories, depending on whether the motion considered occurs "en bloc" or otherwise. The first of these theories, established by Boussinesq and then developed by Serre and Sandover, assumes non-uniform distribution of the velocities-which no longer run parallel-and therefore allows for stream line curvature. Like Sandover, the present authors consider the fiow as having relative motion with respect to a reference system accompanying the wave front, the entrainment velocity being the absolute celerity of the first wave peak.

The apparent rate of flow in the relative motion is constant.

Momentum and specific energy in a cross-section of a rectangular canal are given by equations (1) and (2).

Allowing for the continuity equation and the Eulerian differential equations (4), equations (5) and (6) are obtained by integration of relationships (1) and (2).

If frictional effects and canal slope are assumed to cancel out, momentum and specific energy conservation considerations lead to two second-order differential equations (7) and (11), which when integrated, yield the same equation (10) for the wave profile. The latter equation is also the one for a solitary wave, and enables some of the basic characteristics of this type of wave to be determined.

The maximum rise above the initial water level is given by expression (15).

The total wave volume is given by expression (16).

The apparent rashness of some of the assumptions made for the determination of the secondary undulation characteristics is shown to be partly contradicted by experimental data.

The secondary undulations are assumed to form from a train of solitary waves following each other a finite distance apart. Knowing the local volume of the solitary wave and a celerity relationship, the wave length can be determined, but the authors have preferred relying on a semi-empirical method for this purpose. Inspection of experimental results put forward by several authors shows that, for well-developed undulations, the dimensionless parameter $t_{\epsilon}$ resulting from expression (14) has a roughly constant value, yiz. $t_{\epsilon}=0.956$.

The wave length is thus given by expression (17), based on the above assumption as regards the value of $t_{\epsilon}$. The mean wave height - or weighted height- is then equivalent to half the maximum rise in the water level.

The authors have also considered an approximate approach to the effects of head losses in the light of these assumptions (loss of head varying "stepwise'), the results of which can, however, only give a qualitative idea of the phenomenon. Extreme depth variations with Froude number for relative motion and head loss parameter are shown on Plate 4.

A systematic experimental study has enabled the validity of the assumptions made to be assessed. Comparison between theoretical and experimental data shows satisfactory agreement between both sets for the highest water levels (Fig. 10) and average wave heights (Fig. 12). The dimensionless wave height (Fig. 11) is always between 5 and 10 , usually about 8 .

Figures 7, 8 and 9 show experimental and theoretical wave profile variations for a large initial depth of water and a Froude number of for absolute motion.

A similar investigation has also been carried out for trapezoidal flumes, the theoretical results of which show less pronounced rises in water level than for the rectangular case.

* Maître de conférences, Professeur à l'E.N.S.E.E.H.T.

* Ingénieur diplômé de l'Université de Gdansk. 